Check for updates

Cite this: Chem. Sci., 2019, 10, 10706

๑ All publication charges for this article have been paid for by the Royal Society of Chemistry

Received 16th August 2019

Accepted 23rd September 2019

DOI: $10.1039 / c 9 s c 04100 a$

rsc.li/chemical-science

\section{Dissociation path competition of radiolysis ionization-induced molecule damage under electron beam illumination $\uparrow$}

\author{
Zenghua Cai, (D) a Shiyou Chen (D)*ab and Lin-Wang Wang*c
}

Radiolysis ionization under electron beam illumination induces dissociation and damage of organic and biological molecules; thus, it is impossible to image the related materials by transmission electron microscopy (TEM). To understand the atomistic mechanism of radiolysis damage, we developed a systematical procedure based on real-time time-dependent density functional theory (rt-TDDFT) for simulating the radiolysis damage processes of molecules; this procedure can describe the ionization cross sections of the electronic states and the fast dissociation processes caused by hot carrier cooling and the Auger decay on deep levels. For the radiolysis damage of $\mathrm{C}_{2} \mathrm{H}_{6} \mathrm{O}_{2}$, our simulation unexpectedly showed that there is strong competition among three different dissociation paths, including fast dissociation caused by nonadiabatic cooling of the hot carrier; fast dissociation caused by Auger decay, which induces double ionization and Coulomb explosion; and slow dissociation caused by increased kinetic energy. As the energy of the incident electron beam changes, the time scales of these dissociation paths and their relative contributions to the molecule damage change significantly. These simulation results explain the measured mass spectra of the $\mathrm{C}_{2} \mathrm{H}_{6} \mathrm{O}_{2}$ dissociation fragments and also provide clear competition mechanisms for blocking these dissociation paths in the TEM imaging of organic and biological materials.

\section{Introduction}

The molecular dissociation and damage caused by electron beam illumination is a very important problem in transmission electron microscopy (TEM). ${ }^{1,2}$ From the perspective of electron scattering, ${ }^{2}$ we can categorize electron beam-induced radiation damage into two main classes: (1) elastic scattering, which includes knock-on displacement (atomic displacement), ${ }^{3}$ electrostatic charging ${ }^{4}$ and sputtering; ${ }^{5}$ and (2) inelastic scattering, which includes ionization damage (radiolysis), ${ }^{6}$ heating ${ }^{7}$ and hydrocarbon contamination. ${ }^{8}$ Among these different types of radiation damage, knock-on displacement is the most common elastic scattering damage, while radiolysis is the most common inelastic scattering damage. ${ }^{1,9}$ As discussed in ref. 9, knock-on displacement (where the electron beam gives its momentum and some of its energy to nuclei through Coulomb interactions) is predominant for conducting inorganic materials, ${ }^{10,11}$ while

${ }^{a}$ State Key Laboratory of Precision Spectroscopy, Key Laboratory of Polar Materials and Devices (MOE), Department of Electronics, East China Normal University, Shanghai 200241, China. E-mail: chensy@ee.ecnu.edu.cn

${ }^{b}$ Collaborative Innovation Center of Extreme Optics, Shanxi University, Taiyuan, Shanxi 030006, China

'Materials Sciences Division, Lawrence Berkeley National Laboratory, One Cyclotron Road, Mail Stop 66, Berkeley, CA 94720, USA.E-mail: lwwang@lbl.gov

$\dagger$ Electronic supplementary information (ESI) available. See DOI: 10.1039/c9sc04100a radiolysis (where the material is first ionized by the electron beam) can occur in organic materials and molecules as well as some inorganic systems, such as halides, oxides, hydrides, hydroxides, sulfides and silicates. Furthermore, radiolysis is important in many other processes, including electric discharge and breakdown in high voltage dielectric materials; degradation of polymers and organic electronic devices; excimer lasers; radiation damage of electronic devices in space; radiation chemistry; and biomedicine. ${ }^{12-15}$

However, although radiolysis is highly important, its atomistic mechanism and detailed steps are often not clear. The challenges in studying radiolysis damage reside mainly in the complexity of the process. First, radiolysis-related phenomena can be divided into different situations. Usually, radiolysis involves the ionization of a single electron; ${ }^{1}$ however, a possible related process is that the excited electron is not transported all the way to the vacuum but is transited into higher antibonding states in the system. The ionized or excited electron can come from the valence state ${ }^{1}$ but can also come from the core level of the high energy electron beam. Additionally, in addition to the original ionization due to the high energy external electron beam, the ejected (ionized) electron itself can cause some secondary excitation with lower electron energy. ${ }^{1,9}$ This lower energy electron beam (LEEB) has a larger ionization cross section, although the secondary "beam" intensity will be much smaller compared to the original beam intensity in a TEM 
experiment. However, many experiments have revealed that small molecules can dissociate into different fragments under LEEB illumination. ${ }^{\mathbf{1 6 - 2 4}}$ In this study, we will focus on the original ionization from the valence electron for molecular systems. Although this is only one path among all the possible electron beam damaging scenarios discussed above, it is a common and important path. For a high energy TEM beam, although ionization from the core level is also possible, its cross section is usually much smaller than that of the ionization from the valence electron. Although excitation to the empty antibonding state is also possible, due to the finite density of states of a molecule, its cross section is also smaller for a relatively high energy electron beam; thus, this possibility will be ignored in the current study. Our method is applicable to radiolysis caused by different electron beam energies; however, in order to compare our results with available experiments, we will focus on lower energy electron beam results. The lower energy electron beam results provide more sensitive dependence on the electron energy and thus provide a rich test ground for the calculated results. These low energy results are also relevant to secondary ionization, as discussed above.

In addition to the different situations discussed above, the main challenge for studying radiolysis is the possible complex subsequent steps following the initial ionization, i.e., the ionized molecule can have various dissociation paths depending on the original orbital from which the electron was ejected. As shown schematically in Fig. 1, at least three different processes can be expected: (i) if the ejected electron level is very shallow and close to the highest occupied molecular orbital (HOMO) level, a hot hole carrier will be formed; it will cool with relaxation of the hole to the HOMO, and the energy will be transformed into nuclear kinetic energy. Because the ejected

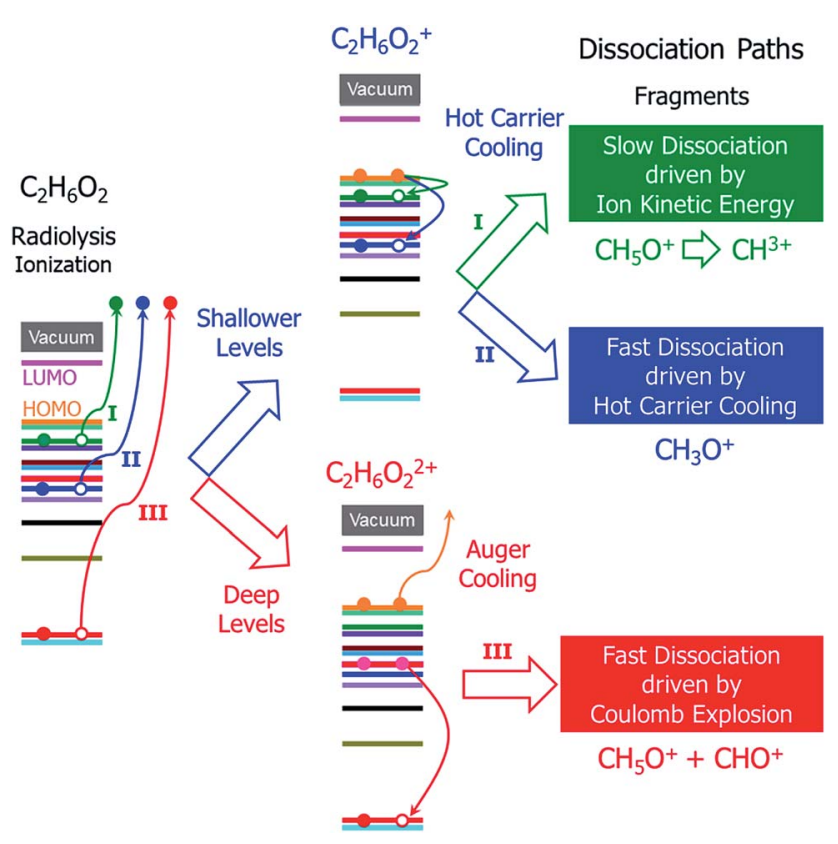

Fig. 1 Schematic of the hot carrier decay processes on different electronic levels and the structural dissociation processes of $\mathrm{C}_{2} \mathrm{H}_{6} \mathrm{O}_{2}$ after radiolysis ionization. electron level is very shallow, the energy from the hot hole relaxation will be small and cannot drive dissociation of the molecule in a short time scale. However, both the hot hole relaxation energy and the reorganization energy of the molecule (the structure of the molecule will be relaxed after an electron is ejected from one occupied state) will be converted into nuclear kinetic energy, which can still dissociate the molecule on a longer time scale; (ii) if the electron is ejected from a level that is slightly lower than the HOMO level, the energy released by hot hole relaxation is higher than in the above situation and will also be converted to nuclear kinetic energy, which may in turn dissociate the molecule; (iii) if the ejected electron level is very deep and much lower than the HOMO level, the large energy from the hot hole relaxation can induce an Auger process with another electron ejected to the vacuum, causing dissociation of the doubly ionized molecule via Coulomb explosion.

It is currently unclear which process is dominant in radiolysis damage and how the energy of the electron beam influences these processes. For example, mass spectroscopy measurements showed that the $\mathrm{C}_{2} \mathrm{H}_{6} \mathrm{O}_{2}$ molecule dissociates into different charged fragments under the illumination of incident electron beams with different kinetic energies; however, it is unknown how $\mathrm{C}_{2} \mathrm{H}_{6} \mathrm{O}_{2}$ is damaged and dissociates into these fragments. To control radiolysis damage, it will be tremendously helpful if we know what process is playing a dominant role and the time scales of these processes. The unclear mechanism of radiolysis damage hinders development in related areas, such as the application of TEM and high voltage dielectric material designs.

$A b$ initio simulation of the radiolysis damage process is an efficient and reliable method for studying atomistic mechanisms and the related detailed steps; however, because the three aforementioned dissociation processes have very different time scales, different $a b$ initio methods are required. Ground state Born-Oppenheimer molecular dynamics (BO-MD) have often been used to simulate knock-on displacement damage ${ }^{25,26}$ however, radiolysis is fundamentally a nonadiabatic process beyond the BO-MD description. ${ }^{27}$ Although there are quantum chemical calculations for nonadiabatic nuclear movement based on the potential energy surface (PES), ${ }^{27}$ they are seldom used to study molecule damage after radiolysis because the related energy potential is too complex to be mapped out in its entirety. In this work, we have developed a practical systematical framework for calculating radiolysis damage, including three modules:

Firstly, we simulated the hot carrier relaxation and the corresponding dissociation during the relaxation process. This was realized by real-time time-dependent density functional theory (rt-TDDFT) simulations. In recent years, we have seen a surge of the use of rt-TDDFT to study various nonadiabatic processes, including ion collisions on materials, ${ }^{28,29}$ dissipative processes in ion irradiation, ${ }^{30}$ carrier-multiplication-induced structural changes $^{31}$ and plasmon excitation in nanowires. ${ }^{32,33}$ It is thus natural to attempt to use rt-TDDFT to describe the hot carrier decay after the initial ionization. Unfortunately, the original rtTDDFT method following Ehrenfest dynamics does not satisfy the detailed balance rule. This causes the electronic degree of 
freedom to be overheated instead of cooled. ${ }^{34}$ In this work, we introduce a method which incorporates detailed balance in rtTDDFT simulations and thus allows study of nonadiabatic hot carrier cooling. Using this modified rt-TDDFT method, we have simulated the hot carrier cooling process in the molecule and the possible fragmentation during this process.

Secondly, the times of the Auger process and the resulting secondary ionization were calculated and compared to the hot hole relaxation time in order to determine which process is dominant. This is necessary if the initial ionized orbital is sufficiently deep and has enough energy to cause secondary ionization. While the nonadiabatic process is induced by electron-phonon interaction, the Auger process is induced by electron-electron interaction. Currently, it is difficult to simulate the Auger process with rt-TDDFT. In this study, we developed an analytical formalism to calculate the Auger cross section with the Fermi-golden rule. We found that whenever energetically possible, the Auger process is extremely fast and is more efficient than nonadiabatic hot carrier decay.

Thirdly, a longer time scale dissociation process was studied according to the nuclear kinetic energies obtained from hot carrier cooling (calculated at the electronic ground state). This occurs if the initial fast nonadiabatic cooling process does not dissociate the molecule and the Auger second ionization does not occur. In $\mathrm{C}_{2} \mathrm{H}_{6} \mathrm{O}_{2}$, we found that this is the case when the initial ionization occurs at the two shallowest orbitals (the HOMO and below the HOMO). In these cases, we did not observe molecule breakdown during the rt-TDDFT simulations. However, thermodynamic energy analysis shows that the molecule can still break down eventually, albeit after overcoming some energy barriers.

Finally, in order to predict the profiles of the initial ionization on different orbital levels, we calculated the cross sections of the ionization rates for different kinetic energies of the illuminating electron beam.

We have used the above procedure to study the mechanisms behind the damage and have compared our results with experimentally observed mass spectroscopy results, especially at relatively low electron beam energies. ${ }^{16}$ We found that after taking into account all the above dissociation processes and ionization cross sections, we can explain some of the qualitative trends in the peaks observed by mass spectroscopy and understand the origins of different charged fragments. This provides previously unknown insights into the molecular breakdown process following ionization radiolysis.

\section{Calculation methodology}

All the first-principles calculations were carried out based on density functional theory (DFT) as implemented by the PWmat code, which uses the plane wave pseudopotential Hamiltonian and runs on graphics processing units (GPU). ${ }^{35,36}$ The generalized gradient approximation (GGA) to the exchange correlation functional in the Perdew-Burke-Ernzerhof $(\mathrm{PBE})^{37}$ form was used with a 50 Ry cutoff energy for the plane wave basis. All the calculations were performed using a $10 \AA \times 10 \AA \times 10 \AA$ cubic cell with one $\mathrm{C}_{2} \mathrm{H}_{6} \mathrm{O}_{2}$ molecule located at the center. A double box formalism (as implemented in PWmat) was used to calculate the Hartree energy with a truncated range Coulomb interaction; therefore, no image interactions existed due to the finite size of the periodic box.

All the modules for calculating the ionization cross sections of the electronic states, the time for double ionization caused by the Auger decay of hot carriers on deep levels, and the thermodynamic dissociation energy costs of singly-ionized and doubly-ionized $\mathrm{C}_{2} \mathrm{H}_{6} \mathrm{O}_{2}$, as well as the rt-TDDFT method, including the detailed balance, were developed based on the PWmat code. The details will be described later.

It is worth commenting on the accuracy of the PBE exchange correlation functional in our DFT calculations. It is well known that PBE-calculated eigenenergies may have significant error when compared with experiments. Here, we chose PBE mostly based on computational efficiency considerations, especially when the plane wave basis set is used. The use of the plane wave basis set is important due to its variational flexibility in describing excited states and ejected electron states. However, the PBE eigenenergy error is mostly a concern for the analytical calculations of the ionization cross section and the Auger effect, where the eigenenergies are used for energy conservation. In the future, more advanced eigenenergy corrections can be used to further correct these energy errors. On the other hand, for rtTDDFT simulations, the results may be more reliable. This is evidenced by the accurate optical absorption spectrum results from the rt-TDDFT simulations for small molecules. In these rtTDDFT simulations, the transition rate is determined more by the total energy change upon a transition than by the eigenenergies. The total energy change is more like the $\Delta$ DFT calculations, which have been shown to be accurate for small molecules. ${ }^{39}$

\section{Results and discussion}

\section{Cross sections of electron ejection on different levels}

Perturbation formalisms exist to calculate the ionization cross sections caused by high velocity incident electrons, such as Bethe theory. ${ }^{38,39}$ For a low energy electron beam (at a few tens of $\mathrm{eV}$, as in experiments), approximated formalisms exist to describe this cross section. One such formalism is the binaryencounter-dipole (BED) model, ${ }^{40}$ which takes into account the acceleration of the incident electron beam caused by nuclear attraction..$^{\mathbf{4 0 4 1}}$ Because this BED model changes to the Bethe theory ${ }^{\mathbf{4 0}}$ at high electron energy, it should also be suitable for a high energy incident electron beam. In Fig. S4 of the ESI, $\dagger$ we show that the ionization cross sections of $\mathrm{C}_{2} \mathrm{H}_{6} \mathrm{O}_{2}$ illuminated by a $100 \mathrm{keV}$ electron beam as calculated by the BED model and the Bethe formula are almost the same. Thus, in our study, the ionization cross sections for all the energy ranges (including the $100 \mathrm{keV}$ ) were calculated with the BED model (as shown in Fig. 3).

In the BED model, an incident electron beam (with initial kinetic energy $T$ ) will be deflected by its Coulomb interaction with a valence electron (at energy $E$ below vacuum) in the molecule and eject the valence electron into the vacuum with 
(a)

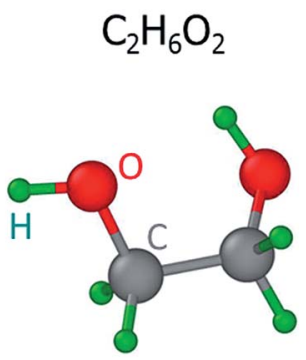

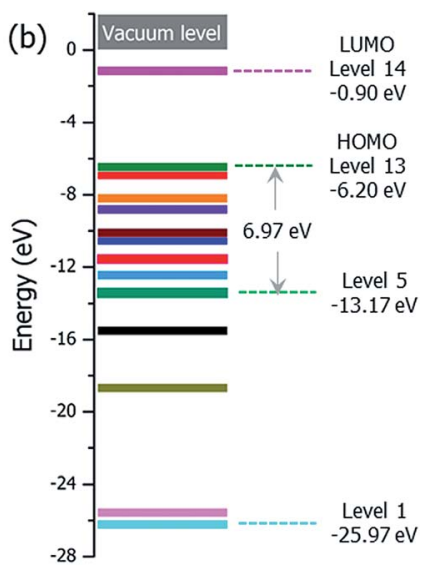

Fig. 2 (a) The ground state structure of the ethylene glycol molecule $\left(\mathrm{C}_{2} \mathrm{H}_{6} \mathrm{O}_{2}\right)$, and (b) the 13 occupied valence orbitals and the lowest unoccupied molecular orbital (LUMO) level. The HOMO level is Level 13, and the LUMO level is Level 14. The vacuum level is set as zero.

kinetic energy $W$. Then, the total cross section $\sigma(t)$ for this ionization process can be calculated as:

$$
\sigma(t)=\frac{S}{t+u+1}\left[D(t) \ln t+\left(2-\frac{N_{i}}{N}\right)\left(\frac{t-1}{t}-\frac{\ln t}{t+1}\right)\right]
$$

in which $S=4 \pi a_{0}^{2} N(R / E)^{2}$ ( $a_{0}$ is the Bohr radius), $t=T / E, u=U /$ $E$ ( $U$ is the average kinetic energy of the ionized valence electron $\left.U \equiv\left\langle\vec{P}^{2}\right\rangle / 2 m_{\mathrm{e}}\right), \vec{P}$ is the momentum operator and $m_{\mathrm{e}}$ is the mass of the electron);

$$
\begin{gathered}
D(t)=N^{-1} \int_{0}^{(t-1) / 2)} \frac{1}{w+1} \frac{\mathrm{d} f(w)}{\mathrm{d} w} \mathrm{~d} w \\
N_{i}=\int_{0}^{\infty} \frac{\mathrm{d} f(w)}{\mathrm{d} w} \mathrm{~d} w
\end{gathered}
$$

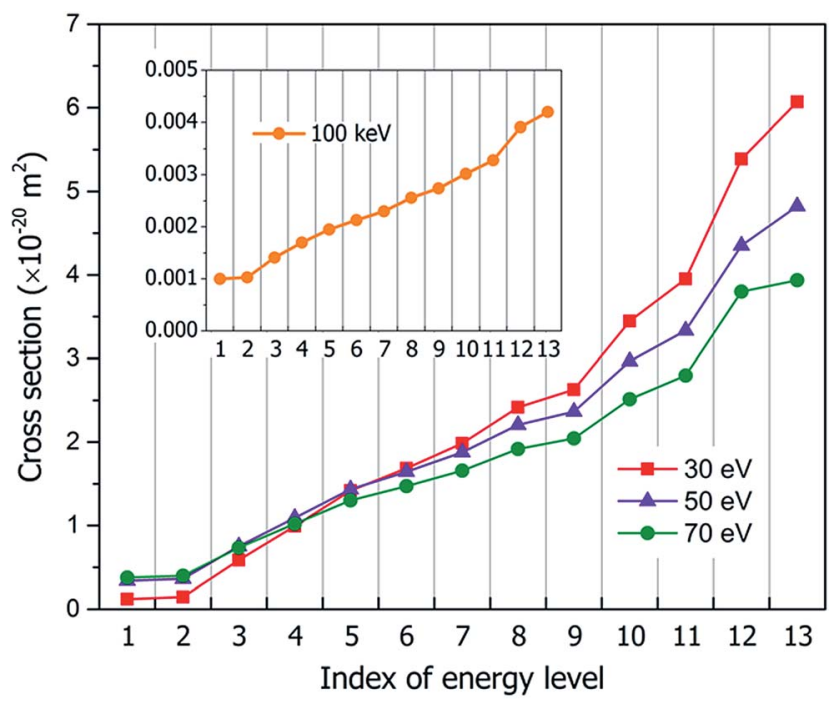

Fig. 3 The calculated ionization cross sections of all 13 occupied levels of $\mathrm{C}_{2} \mathrm{H}_{6} \mathrm{O}_{2}$ under the illumination of electron beams with different incident energies of $30 \mathrm{eV}, 50 \mathrm{eV}, 70 \mathrm{eV}$ and $100 \mathrm{keV}$ (in TEM).

where $N$ is the occupation number of electronic states $(N=2$ for the valence states) and $w=W / E$. For $\frac{\mathrm{d} f(w)}{\mathrm{d} w}$, we take the form of the differential oscillator strength in ref. 38. Using the same notation as above, we have

$$
\begin{gathered}
\frac{\mathrm{d} f(w)}{\mathrm{d} w}=\frac{E(w+1)}{R\left(q a_{0}\right)^{2}} \sum_{\Omega}|\varepsilon(q)|^{2} \\
\varepsilon(q)=\left\langle\phi_{1}\left|\exp \left(i \vec{q} \cdot \vec{r}_{j}\right)\right| \phi_{0}\right\rangle
\end{gathered}
$$

where $\phi_{0}$ represents the wave function of the valence electron to be ionized and $\phi_{1}$ represents the wave function of the ejected valence electron in the vacuum. We used the plane wave function to approximate $\phi_{1}$. We also tested the orthogonalization of $\phi_{1}$ to $\phi_{0}$, and we found that the result does not change significantly. In eqn (4) and (5), $\vec{q}=\left(\vec{K}_{0}-\vec{K}_{1}\right)$ represents the change of the momentum of the electron beam between the incident electron $\left(\vec{K}_{0}\right)$ and the deflected electron $\left(\vec{K}_{1}\right)$. $\Omega$ represents all the possible final plane wave states of the ejected valence electron. As a result, $\sum_{\Omega}|\varepsilon(q)|^{2}$ depends on $w$.

The ground state structure of the $\mathrm{C}_{2} \mathrm{H}_{6} \mathrm{O}_{2}$ molecule is shown in Fig. 2(a), and its molecular orbital energies calculated within DFT (PBE) are shown in Fig. 2(b). There are 13 occupied valence energy levels ( 2 electrons per level). The HOMO level is labeled as Level 13, and the deepest occupied valence level is labeled as Level 1. The valence electron ionization can occur at any one of these energy levels. After the ionization, the neutral $\mathrm{C}_{2} \mathrm{H}_{6} \mathrm{O}_{2}$ molecule becomes the ionized $\mathrm{C}_{2} \mathrm{H}_{6} \mathrm{O}_{2}{ }^{+}$radical with one electron ejected to the vacuum, and all the final fragments come from this ionized radical; this is shown by mass spectroscopy experiments, ${ }^{16}$ where only charged fragments can be observed by mass spectroscopy.

Fig. 3 shows our calculated cross sections for all levels under the illumination of different incident electron beams. The cross sections decrease monotonously from Level 13 to Level 1. This indicates that the electron in the HOMO level is most likely to be ejected into the vacuum (ionized). The predominant reason for this trend is the availability of the final states for the ejected electrons. For a shallow level, the number of the final states is much larger (the sum of $\Omega$ in eqn (4)), which leads to a larger cross section. Furthermore, the ionization cross section decreases as the energy of the incident electron beam increases, except for the very deep levels (Level 1-4) and very low incident electron beam energies, where energy conservation prevents the ionization of the very low energy levels. The smaller ionization cross section for the faster electron beam is due to the very short time in which it passes through the molecule, which prevents the electron from reacting or being ejected.

\section{Molecule dissociation during hot hole carrier cooling}

Following the initial ionization, we performed a rt-TDDFT simulation. A new rt-TDDFT algorithm was implemented. ${ }^{28}$ In this algorithm, the time-dependent electron wave function is expanded on the basis set of adiabatic states. These are the adiabatic states of the TDDFT Hamiltonian $H(t)$ at time $t$, with 
the TDDFT charge density $\rho(r, t)$, instead of the ground state charge density for a given atomic configuration $\{R\}$ at time $t$. This procedure helps to reduce the original high dimensional problem to a small $N$-dimensional problem, where $N$ is the number of adiabatic states included in the expansion. While the $N$ adiabatic state wave functions are updated every $\Delta t(\sim 0.1 \mathrm{fs})$ step, the $N$-dimensional problem is integrated within the $[t, t+$ $\Delta t$ ] interval using a much smaller time step $\mathrm{d} t(\sim 0.1$ attoseconds). However, because this integration is performed in a $N$ $\times N$ Hamiltonian, it can be done quickly. The use of the adiabatic state not only allows us to significantly accelerate the simulation, but also enables us to introduce detailed balance and decoherence. As we know, the direct Ehrenfest dynamics does not satisfy the detailed balance. As a result, it can overheat the electronic system and thus cannot simulate the hot carrier cooling phenomenon.

To restore the detailed balance for the transitions between the adiabatic state $i$ and $j$, we developed an algorithm ${ }^{42}$ based on the occupation number $O(i, t)$ of each adiabatic state $\phi_{i}$; $O(i, t)=\sum_{j} C(i, j, t)^{2} \operatorname{oc}(j), \operatorname{oc}(j)$ is the occupation of the time evolving state $\psi_{j}$ in the rt-TDDFT, and it will not change with time. Note that $\psi_{j}(t)=\sum_{i} C(i, j, t)^{2} \phi_{i}(t)$, where $\psi_{j}$ follows the time-dependent Schrodinger equation $\partial \psi_{j} / \partial t=i H \psi_{j}$. During the time propagation, it is possible to define the change of the adiabatic state occupation as

$$
\begin{aligned}
O(i, t+\mathrm{d} t)= & O(i, t)+\sum_{j} P(i, j, t) O(j, t) \mathrm{d} t \\
& -\sum_{i} P(j, i, t) O(i, t) \mathrm{d} t,
\end{aligned}
$$

where $P(i, j, t)$ represents the charge flow from one adiabatic state $j$ to another adiabatic state $i$ (note: $P(i, j, t) \neq P(j, i, t)$ ). A Boltzmann factor can be applied to this charge flow. If the charge flow is from a higher energy state $j$ to a lower energy state $i$, then no change is needed. Meanwhile, if the charge flow is from a lower energy state $i$ to a higher energy state $j$, a Boltzmann factor $\exp \left(-\left|E_{i}-E_{j}\right| / k T\right)$ can be applied; here, $E_{i}$ and $E_{j}$ are the eigen-energies of the two adiabatic states, $k$ is the Boltzmann constant and $T$ is the temperature. Furthermore, to introduce the decoherence time, the Boltzmann factor is not applied to the instantaneous transition rate $P(i, j, t)$; instead, it is applied to a time-integrated quantity, $P^{\prime}(i, j, t)=\int_{0}^{\infty} P\left(i, j, t-t^{\prime}\right) \exp \left(-t^{\prime} / \tau_{i j}\right) \mathrm{d} t^{\prime}$, where $\tau_{i j}$ is the decoherence time between the two states $i$ and $j$. This $\tau_{i j}$ can be calculated based on the time fluctuations of $E_{i}$ and $E_{j}$, as described in Section 1 of the ESI. $\dagger$ After the Boltzmann factor is applied to $P^{\prime}, O(i, t+\mathrm{d} t)$ will be modified. A special algorithm was developed to change $C(i, j, t)$ in $\psi_{j}(t+\mathrm{d} t)=\sum_{i} C(i, j, t+\mathrm{d} t)^{2} \phi_{i}(t+d t) \quad$ so that the desired renewed occupation $O(i, t+\mathrm{d} t)$ will be reproduced by the modified $C(i, j, t+\mathrm{d} t)$. This treatment of the detailed balance and decoherence time of rt-TDDFT is implemented in the PWmat code. Details about the calculation of the decoherence time are described in Section 1 of the ESI, $\dagger$ and the average of the values calculated using eqn (S1) $\dagger$ is $\tau_{i j}=2$ fs. Our test calculations showed that the exact value of this decoherence time (within a factor of 2) does not affect our results significantly. Note that in our modified Ehrenfest dynamics with the Boltzmann factor and dephasing, our nuclear movement is not on the ground state energy surface but in a mean field excited state energy surface. Although we do not use energy surface hopping, some of the aspects (e.g., detailed balance) do have some commonality with the surface hopping dynamics.

Fig. 4 shows one particular case of the rt-TDDFT simulation where one electron has been ejected from Level 4 at $t=0$. Note that the initial ionization event is not an instantaneous process. Instead, it is a transition from the ground state to a many-body excited state (a higher energy many-body adiabatic state). To represent this higher-energy many-body adiabatic state under the DFT framework, we carried out a constraint DFT calculation at $t=0$ with one electron removed from Level 4 . In other words, $\psi_{j}(t=0)$ in our rt-TDDFT simulations are different from the Kohn-Sham orbitals at the neutral ground state. At $t=0$, the nuclear kinetic energy of the molecule has a temperature of 350 $\mathrm{K}$. As the electronic structure cools from the hot hole state, the released energy is converted to nuclear kinetic energy (which increases its temperature as the time increases), as shown in Fig. 4(a). While the occupation $O(i, t)$ for Level $4(i=4)$ at $t=0$ is 1 , it returns to 2 very quickly (about $35 \mathrm{fs}$ ); then, the occupation of Level 13 (the HOMO level) decreases to 1 (about $100 \mathrm{fs}$ ), indicating that the hole has relaxed to the HOMO level. The full relaxation time of the hot hole on Level 4 is about $100 \mathrm{fs}$, as shown in Fig. 4(b).

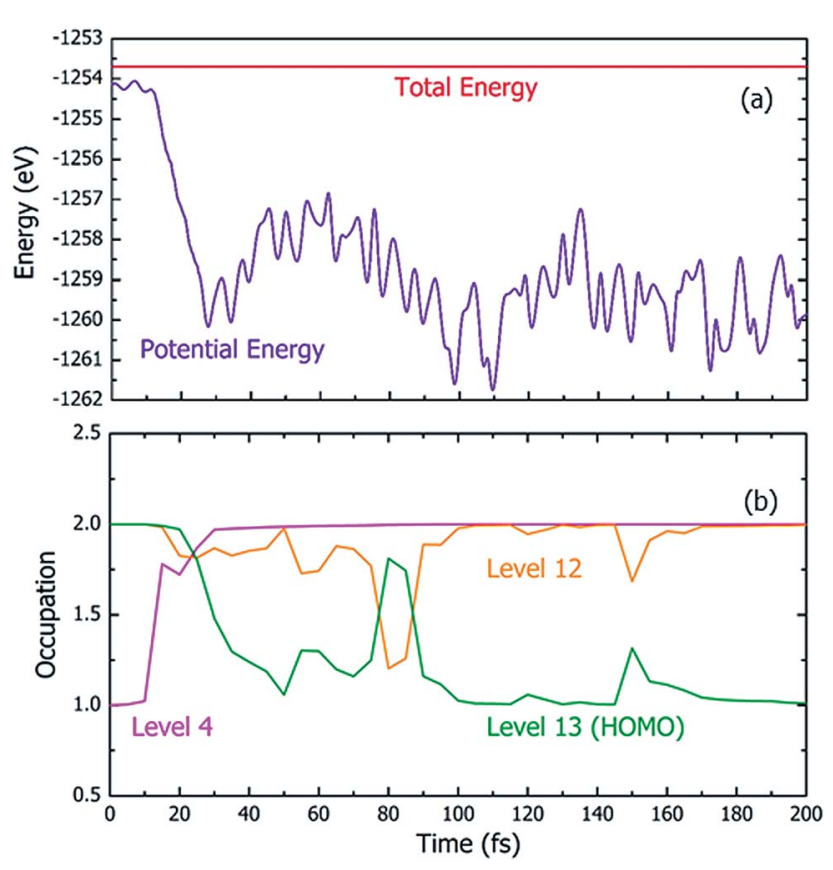

Fig. 4 The changes of (a) the total energy (electronic and nuclear kinetic) and the electronic energy (potential energy), and (b) the electron occupations at different levels (states) in a rt-TDDFT simulation with the detailed balance satisfied. At $t=0$, one electron is ejected from Level 4; therefore, its initial occupation is 1. 
Using the above rt-TDDFT simulations, we systematically studied the hot hole relaxations and the corresponding molecule dissociation processes for all the initial ionization levels (Level 1-13). The total simulation time for each case was $300 \mathrm{fs}$. Although the initial kinetic energy of random atomic movements caused by the $350 \mathrm{~K}$ temperature can modify the results slightly, the overall different initial velocities do not change the results significantly. From the occupation changes during the rt-TDDFT simulation (e.g., in Fig. 4(b)), we can derive the times for the hot hole to cool from the initial ionization level to higher-energy levels. The results for the different initial ionization levels are shown in Fig. 5(a). The cooling times fall in the range from $40 \mathrm{fs}$ to $160 \mathrm{fs}$.

During these simulations, we can also monitor whether the ionized molecule dissociates into different fragments. This is determined by the bond length changes of the molecule. When the atom-atom distance is beyond a certain cutoff and never comes back within a certain period, we can judge that the molecule has dissociated into small fragments, as described in Section 2 of the ESI. $\dagger$ The dissociation times for different ionization levels are also plotted in Fig. 5(a). The corresponding patterns of dissociation are listed in Table 1.

As one can see from Fig. 5(a), remarkably, for most cases, the dissociation time and cooling time are close to each other. After the carrier cools, all its energy has been converted into kinetic energy of the molecule, and the system is in its electronic ground state. This means when the molecule is broken into two fragments during the hot hole cooling, it is the large kinetic

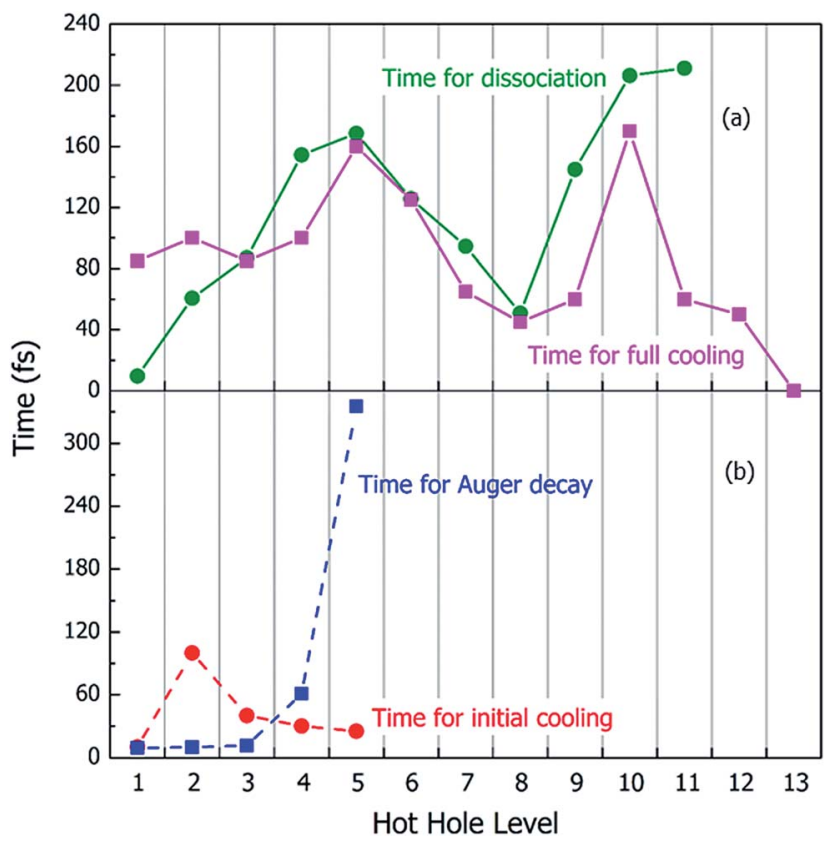

Fig. 5 (a) The times for molecule dissociation and the times for full nonadiabatic cooling of the hot hole carriers (the time to reach thermodynamic equilibrium); (b) the times for Auger decay and the times for initial cooling (the occupation of the initial ionized level increases to 2) of the hot hole carriers after one electron is ejected and a hot hole is formed on different levels of $\mathrm{C}_{2} \mathrm{H}_{6} \mathrm{O}_{2}$. The Auger decay can only occur for Level 1-5.
Table 1 The dissociation of ionized $\mathrm{C}_{2} \mathrm{H}_{6} \mathrm{O}_{2}{ }^{+}$to different fragments for different initial ionization levels according to the rt-TDDFT simulation in $300 \mathrm{fs}$

Initial ionization

level Dissociation to different fragments

$\begin{array}{ll}1 & \mathrm{C}_{2} \mathrm{H}_{6} \mathrm{O}_{2}^{+} \rightarrow \mathrm{C}_{2} \mathrm{H}_{5} \mathrm{O}^{+}\left(\mathrm{CH}_{3} \mathrm{CHOH}^{+}\right)+\mathrm{OH} \\ 2 & \mathrm{C}_{2} \mathrm{H}_{6} \mathrm{O}_{2}^{+} \rightarrow \mathrm{C}_{2} \mathrm{H}_{5} \mathrm{O}^{+}\left(\mathrm{CH}_{2} \mathrm{CH}_{2} \mathrm{OH}^{+}\right)+\mathrm{OH} \\ 3-11 & \mathrm{C}_{2} \mathrm{H}_{6} \mathrm{O}_{2}^{+} \rightarrow \mathrm{CH}_{3} \mathrm{O}^{+}+\mathrm{CH}_{3} \mathrm{O} \\ 12-13 & \text { No dissociation observed }\end{array}$

energy increase (contributed by the hot hole cooling) at the electronic ground state which plays a significant role, rather than the hot hole on a certain level (e.g. the missing of any particular bond orbital). Another important fact is that for all these fast dissociations (ionizations 3-11), we only observed the breaking of the $\mathrm{C}-\mathrm{C}$ bond, as shown in Table 1. This high probability of $\mathrm{C}-\mathrm{C}$ bond breakdown is consistent with the high intensity of $\mathrm{CH}_{3} \mathrm{O}^{+}$fragments observed in the mass spectrum shown in Fig. 6(a).

When the initial ionization levels are Level 12, 13, or 1, 2, the process after ionization is quite different. Firstly, when the ionization occurs at Level 12 or 13 (HOMO level), no dissociation is observed during our simulation for $300 \mathrm{fs}$. The consequence of this will be discussed later in the last subsection of the Results and discussion.

Secondly, when the ionization occurs at Level 1 or 2, the dissociation occurs very quickly, before the hot hole is fully cooled. Especially for Level 1, the dissociation is extremely fast and happens in a few femtoseconds. For both Level 1 and 2, the dissociation patterns are different from those for the other levels. Here, the $\mathrm{C}-\mathrm{O}$ bond is broken, and one $\mathrm{OH}$ group will move away. This results in a $\mathrm{CH}_{2}-\mathrm{CH}_{2}$ group in the case of Level 2. In the case of Level 1, a $\mathrm{H}$ is transferred from one $\mathrm{C}$ to another $\mathrm{C}$, resulting in the formation of a $\mathrm{CH}_{3}$ group. These two

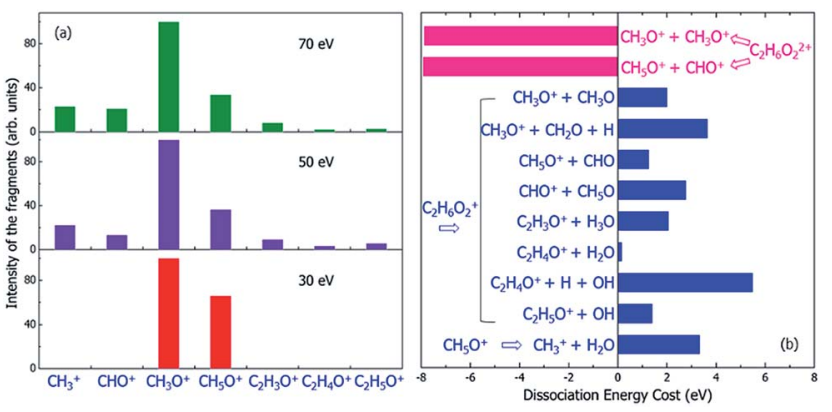

Fig. 6 (a) The experimental mass spectra of the charged fragments from the dissociation of $\mathrm{C}_{2} \mathrm{H}_{6} \mathrm{O}_{2}$ molecule under the illumination of electron beams with different incident energies of 30,50 and $70 \mathrm{eV}$, reproduced from ref. 16. (b) The calculated energy costs for the dissociation of the doubly-ionized $\mathrm{C}_{2} \mathrm{H}_{6} \mathrm{O}_{2}{ }^{2+}$ and singly-ionized $\mathrm{C}_{2} \mathrm{H}_{6} \mathrm{O}_{2}{ }^{+}$into different fragments. Negative dissociation energy means the dissociation is exothermic, while positive energy means the dissociation is endothermic. The dissociation energies were calculated according to the electronic ground state energies of the reaction species. 
processes are shown in Fig. S2(a and b) of the ESI, $\uparrow$ respectively. Note that although the positively charged fragments of the two dissociations can both be denoted as $\mathrm{C}_{2} \mathrm{H}_{5} \mathrm{O}^{+}$, they actually have different structures. According to the rt-TDDFT simulations, we should observe the two different $\mathrm{C}_{2} \mathrm{H}_{5} \mathrm{O}^{+}$fragments experimentally; however, the probability for the experimental observation of $\mathrm{C}_{2} \mathrm{H}_{5} \mathrm{O}^{+}$is quite small according to the mass spectrum shown in Fig. 6(a). To explain this, we investigate the effects of the Auger process in the next section.

\section{Double ionization and dissociation induced by ultra-fast Auger processes}

When the initial ionization occurs at a deep level (Level 1, 2, 3, 4 or 5), the hot hole is deep enough in energy that one electron on a higher energy level can jump down to this level and emit one electron to the vacuum from another level in order to satisfy energy conservation. This is an Auger process. The lifetime $\tau$ of this Auger process can be calculated as $^{43}$

$$
\tau^{-1}=\frac{\Gamma}{\hbar} \sum_{j k l} \frac{|J(j, k, l, m)|^{2}}{(\Delta E)^{2}+(\Gamma / 2)^{2}}
$$

in which

$$
\begin{aligned}
J(j, k, l, m) & =\iint \phi_{\mathrm{j}}^{*}(r) \phi_{k}^{*}\left(r^{\prime}\right) \frac{e^{2}}{\left|r-r^{\prime}\right|} \phi_{l}(r) \phi_{m}\left(r^{\prime}\right) \mathrm{d}^{3} r \mathrm{~d}^{3} r^{\prime} \\
\Delta E & =E(k)-E(m)+E(j)-E(l)
\end{aligned}
$$

$\Gamma$ is a phonon broadening factor, which was set to $30 \mathrm{meV}$ in our calculations. Tests showed that the final result is insensitive to this parameter due to the integration over the final state $l . m$ is the index of the original hot hole level at $t=0, k$ is the index of the level from which one electron jumps down to Level $m$, and $j$ is the index of the level from which one electron is ejected into the vacuum state $l$, as shown in Fig. S3 of the ESI. $\dagger E(k), E(m), E(j)$ and $E(l)$ are the eigenenergies of the four levels in the Auger process. $\hbar$ is the Planck constant. $l$ represents all the possible continuum final levels in the vacuum. While all the bounded orbitals $\phi_{j}, \phi_{k}$, $\phi_{m}$ are provided by the DFT calculations, the emitted state $\phi_{l}$ is approximated by plane-wave functions and then orthogonalized to all the bound states in $\mathrm{C}_{2} \mathrm{H}_{6} \mathrm{O}_{2}$. This orthogonalization is important because the four-center integral of eqn (8) depends sensitively on the orthogonalization between $\phi_{l}$ and $\phi_{m}$. To obtain the total Auger rate by eqn (7), we integrated over all the final states $\phi_{l}$ for the different plane-wave $k$-vectors, while the energy conservation was taken into account by the broadened delta function in eqn (7). No screening was used in calculating the four-center integral of eqn (8) because this is a molecule system, so the dielectric screening is usually rather small.

For each original hot hole Level $m$, multiple Auger channels ( $j$ and $k$ in eqn (7)) can occur. For example, for the hot hole on Level 2, up to 41 possible $(j, k)$ channels of Auger processes were found, and their Auger rates were all calculated. All the possible channels of the Auger processes are listed in Table S1 of the ESI. $\dagger$ The total Auger rate was calculated from eqn (7) by summing up all the channels, as shown in Fig. 5(b). We can see that the Auger lifetime increases dramatically with the initial hot hole level. This is mainly due to the available density of states of the emitted state $l$ for a deep initial level. The overall lifetimes of the Auger processes of Level 1, 2 and 3 are extremely fast, on the time scale of a few femtoseconds. They are all quicker than the initial hot hole cooling process caused by electron-phonon coupling. Here, to compare to the Auger process, we chose the initial cooling times of the hot holes during the rt-TDDFT simulations. This initial cooling time is defined as the time when the initial hot hole level is fully occupied instead of the full relaxation time for the hot hole to relax all the way to the HOMO level. As soon as the original hot hole level is occupied, the corresponding Auger process of that $m$ th level in eqn (7) will no longer be possible. As shown in Fig. 5(b), for Level 1, 2 or 3, the Auger process is faster than the initial cooling time of the hot hole; thus, the Auger process and the secondary ionization (double ionization) will occur. On the other hand, for 4 and 5, the secondary ionization will not occur.

Because the Auger process occurs if a hot hole is formed on Level 1,2 or 3 , the singly-ionized $\mathrm{C}_{2} \mathrm{H}_{6} \mathrm{O}_{2}{ }^{+}$will become the doubly-ionized $\mathrm{C}_{2} \mathrm{H}_{6} \mathrm{O}_{2}{ }^{2+}$. This explains why the predicted fragments $\mathrm{C}_{2} \mathrm{H}_{5} \mathrm{O}^{+}$for Level 1 and 2 in Table 1 (as a result of nonadiabatic decay) are not observed experimentally in Fig. 6(a). $\mathrm{C}_{2} \mathrm{H}_{6} \mathrm{O}_{2}{ }^{2+}$ is not stable; the Coulomb explosion will break it into two fragments. From the pure energy consideration, it can have two different pathways of dissociation. One is into $\mathrm{CH}_{3} \mathrm{O}^{+}+\mathrm{CH}_{3} \mathrm{O}^{+}$, and the other is into $\mathrm{CH}_{5} \mathrm{O}^{+}+\mathrm{CHO}^{+}$. Both dissociation processes are exothermic, as shown by the calculated energy change of the dissociation in Fig. 6(b). The released energies are quite large and are both about $8 \mathrm{eV}$.

The first possible dissociation product of the doubly-ionized $\mathrm{C}_{2} \mathrm{H}_{6} \mathrm{O}_{2}{ }^{2+}$ is $\mathrm{CH}_{3} \mathrm{O}^{+}+\mathrm{CH}_{3} \mathrm{O}^{+}$; thus, only the fragment $\mathrm{CH}_{3} \mathrm{O}^{+}$can be observed (because only charged fragments can be observed by mass spectroscopy), which is the same as the fragment $\mathrm{CH}_{3} \mathrm{O}^{+}$for Level 3-11 in Table 1 . This also explains the high intensity of $\mathrm{CH}_{3} \mathrm{O}^{+}$observed in the mass spectrum shown in Fig. 6(a).

The second possible dissociation product of the doublyionized $\mathrm{C}_{2} \mathrm{H}_{6} \mathrm{O}_{2}{ }^{2+}$ is $\mathrm{CH}_{5} \mathrm{O}^{+}+\mathrm{CHO}^{+}$. However, according to Fig. 6(a), only the fragment $\mathrm{CH}_{5} \mathrm{O}^{+}$is observed, and $\mathrm{CHO}^{+}$is not observed in the mass spectrum when the electron beam energy is $30 \mathrm{eV}$; meanwhile, they are both observed when the electron beam energy is 50 or $70 \mathrm{eV}$. This indicates the $\mathrm{CH}_{5} \mathrm{O}^{+}$in the $30 \mathrm{eV}$ case must come from a different channel instead of from the doubly-ionized $\mathrm{C}_{2} \mathrm{H}_{6} \mathrm{O}_{2}{ }^{2+}$. This is also consistent with the fact that for $30 \mathrm{eV}$, in comparison with the 50 and $70 \mathrm{eV}$ cases, the initial ionization cross sections for Level 1 and 2 are very small, as shown in Fig. 3. Thus, the Auger effect will be small in the case of $30 \mathrm{eV}$. On the other hand, for 50 and $70 \mathrm{eV}$, the Auger effects are large, and we do see both $\mathrm{CH}_{5} \mathrm{O}^{+}$and $\mathrm{CHO}^{+}$in similar amplitudes due to the Auger process.

\section{Slow thermodynamic dissociation of the singly-ionized molecule}

If the initial ionization is on a high-energy level, such as Levels 12 and 13, no fragmentation is observed in our direct rt-TDDFT simulations, as shown in Table 1; meanwhile, there is also not 
enough energy for an Auger process to cause double-ionizationinduced dissociation. However, the ionized $\mathrm{C}_{2} \mathrm{H}_{6} \mathrm{O}_{2}{ }^{+}$can still dissociate because the nuclear kinetic energy increases during the hot hole relaxation and due to the structural reorganization after a hot hole is formed. The increased kinetic energy can overcome some of the dissociation energies, as shown in Fig. 6(b), and cause slow dissociation of the ionized $\mathrm{C}_{2} \mathrm{H}_{6} \mathrm{O}_{2}{ }^{+}$ from the thermodynamic point of view.

In Fig. 6(b), the thermodynamic energy costs for the dissociation of $\mathrm{C}_{2} \mathrm{H}_{6} \mathrm{O}_{2}{ }^{+}$into different fragments are calculated. The energy cost is defined as the change from the energy of the ionized $\mathrm{C}_{2} \mathrm{H}_{6} \mathrm{O}_{2}{ }^{+}$at its ground state (after the hot hole has been cooled to the HOMO level) to the sum of the energies of all the fragments at their ground states (the whole system of all the fragments has one positive charge). The calculated energies of all the fragments in either neutral or +1 charged states are also listed in Table S2. $\dagger$ Compared to the energy of the ionized $\mathrm{C}_{2} \mathrm{H}_{6} \mathrm{O}_{2}{ }^{+}$at its ground state, the total energies of the dissociated fragments are all higher; therefore, the energy costs of all the dissociation paths are positive, in the range from $0.2 \mathrm{eV}$ to $5.5 \mathrm{eV}$.

Because the energy costs are all positive, additional energies are needed to drive these endothermic reactions. If the nuclear kinetic energy is high enough, these dissociations are still possible. This nuclear kinetic energy can come from two sources after an electron is ejected and a hot hole is formed: (i) the nonadiabatic decay and cooling of the hot hole carrier if the original ionization is at Level $1-12$, which can convert the eigenenergy difference between the ionized energy level and the HOMO level to nuclear kinetic energy; and (ii) the reorganization energy of $\mathrm{C}_{2} \mathrm{H}_{6} \mathrm{O}_{2}{ }^{+}$from the original unrelaxed structure of the neutral state (the structure immediately after ionization) to the ground state structure of $\mathrm{C}_{2} \mathrm{H}_{6} \mathrm{O}_{2}{ }^{+}$. Our calculations show that the reorganization energy can be as large as $0.7 \mathrm{eV}$ for Levels 12 and 13. The energy from the hot carrier cooling increases significantly as the initial ionization level becomes deeper. The sum of the two energies increases from $0.7 \mathrm{eV}$ to about $27 \mathrm{eV}$ as the initial ionization level changes from Level 13 to Level 1. Therefore, the increased kinetic energy is close to the energy costs of the dissociation shown in Fig. 6(b); therefore, slow dissociation becomes possible. However, this slow thermally activated dissociation should be in competition with the direct fast nonadiabatic decay-caused dissociation observed in our rt-TDDFT simulation. As shown in Fig. 5(a), in this nonadiabatic process, the time for dissociation is correlated with the time for electronic cooling. This indicates that this dissociation is similar to a ballistic process. Therefore, if this dissociation is possible, the thermally activated slow process will not be competitive. As a result, the thermally activated process may only be relevant for Level 12 and 13 ionizations.

As discussed above, the peaks of $\mathrm{CH}_{5} \mathrm{O}^{+}$and $\mathrm{CHO}^{+}$under illumination of the 50 or $70 \mathrm{eV}$ electron beams result from the dissociation of the doubly-ionized $\mathrm{C}_{2} \mathrm{H}_{6} \mathrm{O}_{2}{ }^{2+}$ after the Auger process; however, only the peak of $\mathrm{CH}_{5} \mathrm{O}^{+}$is observed for the $30 \mathrm{eV}$ electron beam, which indicates that it is not caused by the Auger effect. However, the $\mathrm{CH}_{5} \mathrm{O}^{+}$in the $30 \mathrm{eV}$ case can be explained by the thermally activated slow process for Level 12-
13 ionization through the $\mathrm{C}_{2} \mathrm{H}_{6} \mathrm{O}_{2}^{+} \rightarrow \mathrm{CH}_{5} \mathrm{O}^{+}+\mathrm{CHO}$ process, which has an energy cost of around $1.2 \mathrm{eV}$. The kinetic energy gain from Level 12 ionization is also around $1.2 \mathrm{eV}$, which is sufficient to cause the above dissociation but insufficient to cause the $\mathrm{CH}_{3} \mathrm{O}^{+}+\mathrm{CH}_{3} \mathrm{O}$ dissociation (this process requires about $2.2 \mathrm{eV}$ to occur). Because $\mathrm{CH}_{5} \mathrm{O}^{+}+\mathrm{CHO}$ dissociation requires two $\mathrm{H}$ atoms to transfer from one $\mathrm{C}$ to another $\mathrm{C}$, it will be slow and will only happen in the electronic ground state instead of during the fast hot hole cooling process. When the energy of the electron beam is low (e.g., $30 \mathrm{eV})$, the ionization on the higher energy levels (Level 12 or 13) has larger crosssections, as shown in Fig. 3; therefore, this slow dissociation becomes more dominant, explaining the high $\mathrm{CH}_{5} \mathrm{O}^{+}$peak in Fig. 5(a).

One remaining puzzle is the dissociation into $\mathrm{C}_{2} \mathrm{H}_{4} \mathrm{O}^{+}+\mathrm{H}_{2} \mathrm{O}$. According to our ground state energy calculations, the energy cost of this dissociation is rather small; thus, it should be possible, and the charged $\mathrm{C}_{2} \mathrm{H}_{4} \mathrm{O}^{+}$fragment should be observed. However, no strong peak is observed experimentally for $\mathrm{C}_{2} \mathrm{H}_{4} \mathrm{O}^{+}$, as shown in Fig. 6(a). One possible reason is that due to the high barrier of this reaction, this dissociation path is very improbable, as claimed by Li et al. ${ }^{44}$ using electronic ground state calculations.

Finally, we note that we have yet to find the formation of $\mathrm{CH}_{3}{ }^{+}$in our above analysis; however, the mass spectra show that $\mathrm{CH}_{3}{ }^{+}$is formed under 50 or $70 \mathrm{eV}$ electron beam illumination. We propose that the $\mathrm{CH}_{3}{ }^{+}$arises from a secondary dissociation of $\mathrm{CH}_{5} \mathrm{O}^{+}$into $\mathrm{CH}_{3}{ }^{+}$and $\mathrm{H}_{2} \mathrm{O}$, as first studied in ref. 16. This secondary dissociation requires a dissociation energy of about $3.3 \mathrm{eV}$, as shown in Fig. 6(b); therefore, in the $30 \mathrm{eV}$ case, it cannot occur from $\mathrm{CH}_{5} \mathrm{O}^{+}$, which arises from dissociation due to Level 12 ionization, and very little kinetic energy should remain in $\mathrm{CH}_{5} \mathrm{O}^{+}$. However, large kinetic energy in $\mathrm{CH}_{5} \mathrm{O}^{+}$is possible in the 50 and $70 \mathrm{eV}$ cases because it arises from exothermic $\mathrm{C}_{2} \mathrm{H}_{6} \mathrm{O}_{2}{ }^{2+}$ dissociation, which can release $8 \mathrm{eV}$ kinetic energy, as shown in Fig. 6(b). This agrees with the experimental observation that $\mathrm{CH}_{3}{ }^{+}$only appears in the 50 and $70 \mathrm{eV}$ electron beam cases.

Putting together all the dissociation paths and their competitions, we have tentatively assigned the underlying mechanisms of the main peaks to different dissociation channels (paths) under the illumination of electron beams with different incident energies, as shown in Fig. 7. Note that our results can be verified

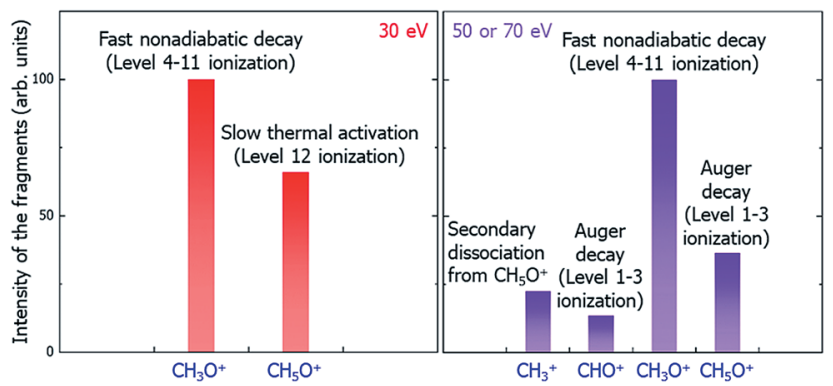

Fig. 7 Schematic of the underlying mechanisms of the main peaks in different electron beam energy situations. 
by future time-resolved mass spectroscopy experiments to distinguish the fast and slow dissociation channels.

\section{Conclusions}

In summary, we have developed a systematic procedure to study the whole radiolysis-induced molecule damaging process. This includes the initial ionization cross-section calculations of different electronic levels under the illumination of electron beams with different incident energies; the nonadiabatic hot carrier cooling calculation following the initial ionization and the associated molecule fragmentation by applying the newly developed rt-TDDFT method, which incorporates the detailed balance; the lifetime calculation of the Auger secondary ionization process; and the calculation of the thermodynamic dissociation energies at the final electronic ground state. Taking the dissociation processes of $\mathrm{C}_{2} \mathrm{H}_{6} \mathrm{O}_{2}$ molecule after the initial radiolysis ionization caused by LEEB illumination as an example, through systematical calculations using these methods and comparing the time scales and competitiveness of different dissociation paths, we have revealed the underlying mechanisms of the peaks of different charged fragments in the observed mass spectra. We are not at the level of predicting the quantitative value of the amplitude of each peak; however, we can tentatively assign the origin of each peak and predict the trend of its amplitude as a function of the illuminating electron beam energy. We found three categories of dissociation paths: the fast and ballistic dissociation during hot hole carrier cooling $\left(\mathrm{CH}_{3} \mathrm{O}^{+}\right)$; the fast Coulomb explosion caused by secondary ionization due to the Auger effect $\left(\mathrm{CH}_{5} \mathrm{O}^{+}, \mathrm{CHO}^{+}\right.$for the 50 and $70 \mathrm{eV}$ electron beams); the slow dissociation driven by the kinetic energy at the electronic ground state $\left(\mathrm{CH}_{5} \mathrm{O}^{+}\right.$for the $30 \mathrm{eV}$ electron beam); and the secondary fragment decay from the initial decay fragments $\left(\mathrm{CH}_{3}{ }^{+}\right.$decayed from $\mathrm{CH}_{5} \mathrm{O}^{+}$for the 50 and $70 \mathrm{eV}$ electron beams). Our results explain all the main peaks in the experimentally observed mass spectra and their relative amplitudes and qualitative trends as a function of the electron beam energy. Our work demonstrates that it is now possible to use $a b$ initio calculations to understand the complex underlying mechanisms of molecule damage under electron beam illumination in TEM experiments. The insights provided by the current work and the identification of different dissociation paths can help us to find solutions to mitigate the molecule damage problem in TEM experiments as well as in many other applications. The methods developed here can also be adopted for the study of defect formation or bond breaking processes in bulk materials under electron beam illumination.

\section{Conflicts of interest}

There are no conflicts to declare.

\section{Acknowledgements}

L. W. W. was supported by the US Department of Energy, Office of Science, Office of Basic Energy Sciences, Materials Sciences and Engineering Division under contract no. DE-AC02-05-
CH11231 within the in situ TEM program (KC22ZH). H. C. and S. C. were supported by the National Natural Science Foundation of China (NSFC) under grant No. 61722402, 61574059 and 91833302, the National Key Research and Development Program of China (2016YFB0700700), Shanghai Academic/ Technology Research Leader (19XD1421300), Fok Ying Tung Education Foundation (161060), the Fundamental Research Funds for the Central Universities and CC of ECNU. This work used the resources of the National Energy Research Scientific Computing Center.

\section{Notes and references}

1 R. F. Egerton, Ultramicroscopy, 2013, 127, 100.

2 R. F. Egerton, P. Li and M. Malac, Micron, 2004, 35, 399.

3 O. Ugurlu, J. Haus, A. A. Gunawan, M. G. Thomas, S. Maheshwari, M. Tsapatsis and K. A. Mkhoyan, Phys. Rev. B: Condens. Matter Mater. Phys., 2011, 83, 113408.

4 L. Reimer, U. Golla, R. Böngeler, M. Kässens, B. Schindler and R. Senkel, Optik, 1992, 92, 14.

5 L. E. Thomas, Ultramicroscopy, 1985, 18, 173.

6 R. Henderson and R. M. Glaeser, Ultramicroscopy, 1985, 16, 139.

7 M. Liu, L. Xu and X. Lin, Scanning, 1994, 16, 1.

8 J. J. Hren, Barriers to AEM: Contamination and Etching, in Introduction to Analytical Electron Microscopy, Springer US, 1979.

9 R. F. Egerton, Microsc. Res. Tech., 2012, 75, 1550.

10 J. C. Meyer, C. O. Girit, M. F. Crommie and A. Zettl, Nature, 2008, 454, 319.

11 A. Hashimoto, K. Suenaga, A. Gloter, K. Urita and S. Iijima, Nature, 2004, 430, 870.

12 T. D. Märk and G. H. Dunn, Electron impact ionization, Springer Science \& Business Media, 2013.

13 L. G. Christophorou, Electron-molecule interactions and their applications, Academic Press, 2013.

14 L. C. Pitchford, B. V. McKoy, A. Chutjian and S. Trajmar, Swarm studies and inelastic electron-molecule collisions, Springer Science \& Business Media, 2012.

15 V. Garner, Biomedical applications/Gas chromatography-mass spectrometry, Academic Press, 2000.

16 M. Y. Mykyta, L. G. Romanova, A. M. Zavyilopulo and O. B. Shpenik, Ukr. J. Phys., 2011, 56, 116.

17 D. Bouchiha, J. D. Gorfinkiel, L. G. Caron and L. Sanche, J. Phys. B: At., Mol. Opt. Phys., 2007, 40, 1259.

18 A. N. Zavilopulo, F. F. Chipev and L. M. Kokhtych, Nucl. Instrum. Methods Phys. Res., Sect. B, 2005, 233, 302.

19 R. Rejoub, C. D. Morton, B. G. Lindsay and R. F. Stebbings, J. Chem. Phys., 2003, 118, 1756.

20 J. E. Hudson, M. L. Hamilton, C. Vallance and P. W. Harland, Phys. Chem. Chem. Phys., 2003, 5, 3162.

21 L. G. Christophorou and J. K. Olthoff, J. Phys. Chem. Ref. Data, 2000, 29, 267.

22 R. Völpel, G. Hofmann, M. Steidl, M. Stenke, M. Schlapp, R. Trassl and E. Salzborn, Phys. Rev. Lett., 1993, 71, 3439.

23 F. L. Arnot, Nature, 1932, 129, 617.

24 L. Sanche, Mass Spectrom. Rev., 2002, 21, 349. 
25 S. T. Skowron, I. V. Lebedeva, A. M. Popov and E. Bichoutskaia, Nanoscale, 2013, 5, 6677.

26 T. Susi, J. Kotakoski, R. Arenal, S. Kurasch, H. Jiang, V. Skakalova, O. Stephan, A. V. Krasheninnikov, E. I. Kauppinen, U. Kaiser and J. C. Meyer, ACS Nano, 2012, 6, 8837.

27 B. F. E. Curchod and T. J. Martínez, Chem. Rev., 2018, 118, 3305.

28 Z. Wang, S.-S. Li and L.-W. Wang, Phys. Rev. Lett., 2015, 114, 063004.

29 G. Bi, J. Kang and L.-W. Wang, Phys. Chem. Chem. Phys., 2017, 19, 9053.

30 R. Ullah, E. Artacho and A. A. Correa, Phys. Rev. Lett., 2018, 121, 116401.

31 J. Bang, Y. Y. Sun, X. Q. Liu, F. Gao and S. B. Zhang, Phys. Rev. Lett., 2016, 117, 126402.

32 F. Ding, E. B. Guidez, C. M. Aikens and X. Li, J. Chem. Phys., 2014, 140, 244705.

33 B. Peng, D. B. Lingerfelt, F. Ding, C. M. Aikens and X. Li, J. Phys. Chem. C, 2015, 119, 6421.
34 W. H. Miller and S. J. Cotton, J. Chem. Phys., 2015, 142, 131103.

35 W. Jia, J. Fu, Z. Cao, L. Wang, X. Chi, W. Gao and L.-W. Wang, J. Comput. Phys., 2013, 251, 102.

36 W. Jia, Z. Cao, L. Wang, J. Fu, X. Chi, W. Gao and L.-W. Wang, Comput. Phys. Commun., 2013, 184, 9.

37 J. P. Perdew, K. Burke and M. Ernzerhof, Phys. Rev. Lett., 1996, 77, 3865.

38 M. Inokuti, Rev. Mod. Phys., 1971, 43, 297.

39 M. Inokuti, Y. Itikawa and J. E. Turner, Rev. Mod. Phys., 1978, 50, 23.

40 Y.-K. Kim and M. E. Rudd, Phys. Rev. A: At., Mol., Opt. Phys., 1994, 50, 3954.

41 Y. K. Kim, W. Hwang and M. E. Rudd, AIP Conf. Proc., 1996, 381, 93.

42 J. Kang and L.-W. Wang, Phys. Rev. B, 2019, 99, 224303.

43 L.-W. Wang, M. Califano, A. Zunger and A. Franceschetti, Phys. Rev. Lett., 2003, 91, 056404.

44 Y. Li and T. Baer, J. Phys. Chem. A, 2002, 106, 8658. 